\title{
Self-consistent nonperturbative anomalous dimensions
}

\author{
R Delbourgo $\dagger$ \\ † School of Mathematics and Physics, University of Tasmania, Box 252-21, \\ AUSTRALIA 7001 \\ E-mail: Bob.Delbourgo@utas.edu.au
}

\begin{abstract}
A self-consistent treatment of two and three point functions in models with trilinear interactions forces them to have opposite anomalous dimensions. We indicate how the anomalous dimension can be extracted non-perturbatively by solving and suitably truncating the topologies of the full Dyson-Schwinger set of equations. The first step requires a sensible ansatz for the full vertex part, which conforms to first order perturbation theory at least. We model this vertex to obtain typical transcendental relations between anomalous dimension and coupling constant $g$ which coincide with known results to order $g^{4}$.
\end{abstract}

Submitted to: J. Phys. A: Math. Gen.

PACS numbers: $11.10 \mathrm{Gh}, 11.10 \mathrm{Jj}, 11.10 \mathrm{Kk}, 11.15 \mathrm{Tk}$ 


\section{Non-perturbative equations}

Renormalizable quantum field theories like QED, QCD or pseudoscalar Yukawa theory are characterised by anomalous dimensions which determine the asymptotic properties of the Green functions as all momenta are scaled. Specifically the anomalous dimension $\gamma$ of a quantum field is determined through the asymptotic behaviour of the propagator $\left(p^{2}\right)^{-1-\gamma}$ when $p^{2} \rightarrow \infty$. The calculation of $\gamma$ as a power series in the renormalized coupling constant $g$, arising in the trilinear interaction Lagrangian, has occupied much time and effort and is known up to order $g^{8}$ for certain models. It would be nice if one could calculate $\gamma$ to all orders of $g$ by summing subclasses of diagrams, corresponding to particular topologies, without excessive effort. This paper is devoted to outlining a method by which this might be achieved. It is based on conformal scaling of Green functions at asymptotic momenta, with anomalous dimensions providing the self-regulation of the field theory [1, 2] in question. Such a proposal makes eminently good sense at a zero $g=g^{*}$ of the beta function on the positive real line and we simply assume that this applies in all that follows. However it must be pointed out that for the models considered later there is no indication of such a zero and with its corresponding coupling constant.

The basic idea behind the method is to eliminate the renormalization constants as far as it is possible and to write the renormalized Green functions in terms of one another as a power series in the coupling constant with ever more complicated topologies. This produces a set of self-consistent Schwinger-Dyson equations of the full propagator in terms of itself and the proper vertex part, though there exist infinitely many different topological terms in the skeleton expansion of course. In the end one is forced to truncate these topological contributions, but in any case it is rather easy to show (see below) that the only self-consistent behaviour is one where the anomalous vertex and propagator dimensions are oppositely related. The equations in principle then yield an equation for the anomalous dimensions in terms of the renormalized coupling constant. In the past it has proved relatively simple to sum over particular topologies where the vertex function remains undressed, but in this paper we shall emphasize the importance of considering the full three-point vertex (not just in some kinematic limit) when studying these equations; this is the novel aspect of our work and by this means one may hope to improve on the rainbow [3, 4], ladder and chain [5] approximations of diagram sets the favourite ones studied thus far and with great aplomb by Broadhurst and Kreimer.

To illustrate what is involved, consider the case of the renormalizable $g \sigma \phi^{\dagger} \phi$ interaction in six dimensions, rather than $g \phi^{3} / 6$ ! theory [6], ignoring triple $\sigma$ interactions for the moment. Including renormalization constants, the equation for the $\phi$ and $\sigma$ propagators, $\Delta_{\phi}$ and $\Delta_{\sigma}$ respectively, expressed in terms of full Green functions, in the massless case reads

$$
\begin{aligned}
\Delta_{\phi}^{-1}(p) & =Z_{\phi} p^{2}-i g^{2} Z_{g} \int \bar{d}^{6} k \Gamma(p, p+k, k) \Delta_{\phi}(k) \Delta_{\sigma}(p+k) \\
& =Z_{\phi} p^{2}-i g^{2} \int \bar{d}^{6} k \Gamma(p, p+k, k) \Delta_{\phi}(k) \Delta_{\sigma}(p+k) \Gamma(p, p+k, k)
\end{aligned}
$$


Proper vertex

$$
\begin{gathered}
-g^{4} \int \bar{d}^{6} k \bar{d}^{6} q \Gamma(p, p+k, k) \Delta_{\phi}(k) \Delta_{\sigma}(p+k) \Delta_{\phi}(p+q) \Delta_{\sigma}(q) \Gamma(p, p+q, q) \\
\Gamma(q, k, q-k) \Delta_{\phi}(k-q) \Gamma(p+k, q+k, k-q) \cdots \\
\Delta_{\sigma}^{-1}(p)=Z_{\sigma} p^{2}-i g^{2} Z_{g} \int \bar{d}^{6} k \Gamma(p, p+k, k) \Delta_{\phi}(k) \Delta_{\phi}(p+k) \\
=Z_{\sigma} p^{2}-i g^{2} \int \bar{d}^{6} k \Gamma(p, p+k, k) \Delta_{\phi}(k) \Delta_{\phi}(p+k) \Gamma(p, p+k, k) \\
-g^{4} \int \bar{d}^{6} k \bar{d}^{6} q \Gamma(p, p+k, k) \Delta_{\phi}(k) \Delta_{\phi}(p+k) \Delta_{\phi}(p+q) \Delta_{\phi}(q) \Gamma(p, p+q, q) \\
\Gamma(q, k, q-k) \Delta_{\sigma}(k-q) \Gamma(p+k, q+k, k-q) \cdots
\end{gathered}
$$

For each field, we know that in renormalized perturbation theory the sum of the series will produce the large $p$ scaling behaviour, $\Delta^{-1}(p) \simeq c p^{2}\left(-p^{2} / \mu^{2}\right)^{\gamma}$, where $\gamma$ is given as a series in $g^{2}$, which can be computed $\ddagger$ order by order in perturbation theory, albeit with greater and greater pain as the power of $g^{2}$ rises. The purpose of this paper is to look for relations between $\gamma$ and $g^{2}$, which correspond to particular truncations of various topological contributions to the self-energy; such relations are normally given by a transcendental equation that can be found via the Dyson-Schwinger equations. But how do we find them by suitably manipulating eqs (1) and (2)? For the moment neglect the first term on the right, which involves the renormalization constant and seems to scale as $p^{2}$; the remaining terms have the form

$$
g^{2} F_{2}\left(p, \mu, \gamma\left(g^{2}\right)\right)+g^{4} F_{4}\left(p, \mu, \gamma\left(g^{2}\right)\right)+\cdots
$$

and we must ask how they sum up to the scaling form on the left-hand side. It is possible, but extremely unlikely, that each of these terms behaves as $p^{2}$ aside from logarithms and that each contains an infinity (which is subtracted off by the $Z$-factor on the right) and that they somehow combine to produce the anomalous scaling; if that were true there would be little point in using the skeleton expansion as a means of transcending perturbation theory. A more likely scenario, which we shall assume hereafter, is that each of the contributions $F_{N}$ scale in exactly the same way. Then it is not hard to work out what must be the scaling behaviour of the proper vertex $\Gamma$ to produce $\left(p^{2}\right)^{1+\gamma}$ at large $p^{2}$. One easily establishes that

$$
\Gamma(\lambda p, \lambda(p+k), \lambda k) \sim \lambda^{2 \gamma_{\Gamma}} \Gamma(p, p+k, k) ; \quad \gamma_{\Gamma}=\gamma_{\phi}+\gamma_{\sigma} / 2
$$

will ensure that all of the topological contributions $F_{N}$ to the two-point function yield the same scaling as the external momentum $p \rightarrow \infty$. [Without losing too much generality one may fix the vertex function $\Gamma=1$ either at the symmetrical Euclidean point $p^{2}=(p+k)^{2}=k^{2}=-\mu^{2}$ or at zero-momentum transfer $k=0, p^{2}=(p+k)^{2}=-\mu^{2}$, but that still leaves vast freedom in the dynamics of $\Gamma$ in $(2)$, through its dependence on the momentum ratios.] As for justifying why we drop the renormalization terms $Z p^{2}$, we note that the wave function renormalization constant may be generally defined

$\ddagger$ We anticipate that the constant $c$ appearing in $\Delta$ behaves like unity when $\gamma \rightarrow 0$; this is because the imaginary part of $\Gamma(1+\gamma) /\left(p^{2}+i \epsilon\right)^{1+\gamma}$ equals $-\pi \theta\left(-p^{2}\right) / \Gamma(-\gamma)\left(p^{2}\right)^{1+\gamma}$ and this generalised function reduces to $-\pi \delta\left(p^{2}\right)$ for the free case $\gamma=0$. 
by $Z^{-1} \equiv \lim _{p^{2} \rightarrow \infty} p^{2} \Delta(p)$ and then $Z$ can well vanish $\S$ for negative $\gamma$, in which case dropping it from the self-consistent but nonperturbative Schwinger-Dyson equations is not entirely absurd in the asymptotic limit.

Specifically, let us contemplate the situation where one the fields, say $\sigma$, remains undressed (some sort of quenched approximation in which closed $\phi$-loop graphs are disregarded, signifying that induced multisigma interactions can be dropped). Thus its corresponding $Z_{\sigma}=1, \gamma_{\sigma}=0$, so the scaling of $\Gamma$ just becomes tied to the asymptotic behaviour of the field $\phi$ : they are inverse to one another. This connection is unsurprising especially in QED where the Ward identity $\Gamma(p, p, 0)=\partial \Delta^{-1} / \partial p$ at zero photon momentum, already indicates that the scaling properties of the charged field and the soft vertex are intimately tied (' $Z_{1}=Z_{2}$ ' in a perturbative context). In Section 2 we summarize what is known about the vertex function in $\sigma \phi^{\dagger} \phi$ and Yukawa theory, the two models upon which we focus. There we look for a nonperturbative version of $\Gamma$ which reduces to first order perturbation theory, with the correct singularities. Then in Section 3 we show how this can be used to determine the relation between $\gamma$ and $g^{2}$ even in the simplest kind of truncation encompassing the chain and rainbow summations with various model vertex functions.

\section{Structure of the proper vertex}

\subsection{Lowest order structure}

One of the most elementary exercises in quantum field theory is to work out the first order corrections to the propagator and vertex part, where we normally encounter infinities that must be renormalized. Working in $2 \ell$ dimensions (for isolating the infinities) leads one to the typical expressions for the self-energy $\Sigma$ and proper vertex correction $\Lambda$,

$$
\begin{gathered}
\Sigma(p)=c_{2} g^{2} \iint_{0}^{1} d x d y \frac{\delta(x+y-1) \Gamma(2-\ell)}{\left[p^{2} x y-m_{1}^{2} x-m_{2}^{2} y\right]^{2-\ell}} \\
\Lambda\left(p_{1}, p_{2}, p_{3}\right)=c_{3} g^{2} \iiint_{0}^{1} d x d y d z \frac{\delta(x+y+z-1) \Gamma(3-\ell)}{\left[p_{1}^{2} y z+p_{2}^{2} z x+p_{3}^{2} x y-m_{1}^{2} x-m_{2}^{2} y-m_{3}^{2} z\right]^{3-\ell}},
\end{gathered}
$$

where $\ell \rightarrow n=2$ or 3 in the integer limit and $c_{i}$ are symmetry factors including $1 /(4 \pi)^{\ell}$. Subtraction of the pole terms by renormalization constants produces logarithms of the denominators of each of the right hand sides, normalized at some mass scale $\mu$, which is how dimensional transmutation and the renormalization prescription enters. One can recognize the resulting expressions as the first terms of an expansion - which strictly makes sense at a zero of the beta-function when we proceed to the nonperturbative regime - of the Green functions in powers of $g^{2}$; for example for the massless two-point function, where the logarithmic term is easily computed, we end up straightforwardly $\S$ The condition $Z=0$ applies to composite particles and agrees with this observation, but becomes problematic for positive $\gamma$. 
with

$$
\Delta^{-1}(p)=p^{2}+c_{2} g^{2} p^{2} \log \left(-p^{2} / \mu^{2}\right)+\cdots \simeq p^{2}\left(-p^{2} / \mu^{2}\right)^{\gamma}
$$

in keeping with $\gamma=c_{2} g^{2}+\cdots$. For the three-point function, renormalized at zero $\sigma$ momentum, the logarithm is actually a dilogarithm, being given by the integral

$$
\begin{aligned}
-\Lambda & =\int_{0}^{1} \int_{0}^{1} \int_{0}^{1} d x d y d z \delta(x+y+z-1) \log \left(\frac{p_{1}^{2} y z+p_{2}^{2} z x+p_{3}^{2} x y}{-\mu^{2}(y z+z x)}\right) \\
& =\frac{c_{3} g^{2}}{2} \int_{0}^{1} \sigma d \sigma \int_{-1}^{1} d \tau \log \left[\left(\frac{-p_{1}^{2}}{2 \mu^{2}}\right)(1-\tau)+\left(\frac{-p_{2}^{2}}{2 \mu^{2}}\right)(1+\tau)-\frac{p_{3}^{2} \sigma\left(1-\tau^{2}\right)}{4 \mu^{2}(1-\sigma)}(4)\right.
\end{aligned}
$$

whose singularities characterize the 'triangle' graph. It simplifies to pure logarithms when $p_{3}^{2} \rightarrow 0$, being proportional to

$$
\frac{1}{2}\left[\log \left(\frac{p_{1}^{2} p_{2}^{2}}{\mu^{4}}\right)-2+\frac{p_{2}^{2}+p_{1}^{2}}{p_{2}^{2}-p_{1}^{2}} \log \left(\frac{p_{2}^{2}}{p_{1}^{2}}\right)\right],
$$

which one should note is the order $\gamma$ term of the expression

$$
\frac{\mu^{2}}{(1+\gamma)\left(p_{1}^{2}-p_{2}^{2}\right)}\left[\left(-\frac{p_{2}^{2}}{\mu^{2}}\right)^{\gamma+1}-\left(-\frac{p_{1}^{2}}{\mu^{2}}\right)^{\gamma+1}\right]
$$

This bears an uncanny resemblance to the ratio $\left[\Delta^{-1}\left(p_{2}\right)-\Delta^{-1}\left(p_{1}\right)\right] /\left(p_{2}^{2}-p_{1}^{2}\right)$ that one encounters when "solving" for the longitudinal 3-point vertex in gauge theories; we'll come back to this point presently. In any case it strongly suggests that the nonperturbative form of the vertex possessing triangular topology exponentiates to

$$
\Gamma\left(p_{1}, p_{2}, p_{3}\right)=2 \iiint_{0}^{1} d x d y d z \rho \delta(x+y+z-1)\left[\frac{p_{1}^{2} y z+p_{2}^{2} z x+p_{3}^{2} x y}{-\mu^{2}(y z+z x)}\right]^{\gamma_{\Gamma}},
$$

where the (symmetric in $y, z)$ spectral function $\rho(x, y, z)$ equals 1 up to first order in $g^{2}$ and $\gamma_{\Gamma}=-c_{3} g^{2} / 2$. If we restrict ourselves to such topology, neglect the dressing of $\sigma$ and reinsert (4) into (1), this will produce a self-consistent equation for $\gamma_{\phi}$ in terms of $g^{2}$, as we already know that uniform scaling of $F_{N}$ requires $\gamma_{\Gamma}=\gamma_{\phi}$ when $\sigma$ is quenched.

The Feynman parametric form of $\Lambda$ in the limit as $p_{3}^{2}=0$, (but not $p_{3}=0$ ) is very suggestive of another Lehmann-like representation which one encounters in the gauge technique. Thus by changing variable to $w^{2}=m_{1}^{2} / y+m_{2}^{2} / x$ in the self-energy, one may convert from Feynman to dispersive form:

$$
\Sigma(p)=\int_{\left(m_{1}+m_{2}\right)^{2}}^{\infty} d w^{2} \sigma\left(w^{2}\right) /\left(p^{2}-w^{2}\right) .
$$

In the particular case that $m_{1}=m_{2}=m$ for the vertex function, which is often true, one can make a similar conversion in $\Lambda$ as $p_{3}^{2} \rightarrow 0$ :

$$
\begin{aligned}
& \Lambda \propto \int_{0}^{1} d x \int_{-x}^{x} d u \frac{\frac{1}{2} \Gamma(3-\ell)}{\left[\frac{1}{2}\left(p_{1}^{2}+p_{2}^{2}\right) x(1-x)+\frac{1}{2}\left(p_{1}^{2}-p_{2}^{2}\right) u(1-x)-m^{2} x\right]^{3-\ell}} \\
= & \int_{0}^{1} \frac{\Gamma(2-\ell) d x}{\left(p_{2}^{2}-p_{1}^{2}\right)(1-x)}\left[\frac{1}{\left(p_{1}^{2} x(1-x)-m^{2} x\right)^{2-\ell}}-\frac{1}{\left(p_{2}^{2} x(1-x)-m^{2} x\right)^{2-\ell}}\right],
\end{aligned}
$$




$$
\text { and closely resembling } \frac{\Sigma\left(p_{1}^{2}\right)-\Sigma\left(p_{2}^{2}\right)}{p_{2}^{2}-p_{1}^{2}}=\int \frac{d w^{2} \sigma\left(w^{2}\right)}{\left(p_{1}^{2}-w^{2}\right)\left(p_{2}^{2}-w^{2}\right)}
$$

Thus we anticipate that the vertex possesses a simplified dispersive-like representation when one of the momenta is lightlike, and this property might prove rather useful. Further progress may need additional truncation of the skeleton expansion and other practical simplifications.

\subsection{Perturbation theory}

These remarks apply to all theories with basic trilinear interactions. Before looking at nonperturbative aspects of the models $g\left(\sigma \phi^{\dagger} \phi\right)_{6 D}$ and $\left(g \bar{\psi} \gamma_{5} \psi \phi\right)_{4 D}$, let us first note the order $g^{2}$ results for $c_{2}, c_{3}$ since they supply helpful weak-coupling limits for the two and three point functions involving massless fields $\phi, \sigma$ and $\psi$.

In $g\left(\phi^{\dagger} \phi \sigma\right)_{6 D}$ one trivially finds that

$$
\Sigma(p)=\frac{g^{2}}{p^{4}}\left(\frac{-p^{2}}{4 \pi}\right)^{\ell} \frac{\Gamma(2-\ell) \Gamma(\ell-1) \Gamma(\ell-1)}{\Gamma(2 \ell-2)}
$$

where the limit $\ell \rightarrow 3$ must be taken. Renormalizing at $p^{2}=-\mu^{2}$, one ends up with

$$
\Sigma(p)=-\frac{g^{2} p^{2}}{6(4 \pi)^{3}} \ln \left(-\frac{p^{2}}{\mu^{2}}\right)
$$

Likewise the vertex correction is just expression (4), with $c_{3}=1 /(4 \pi)^{3}$. It may be expressed [7] as an Appell function, but better still, it can be converted into a symmetrical sum of three hypergeometric functions in arbitrary dimensions [8]; we will utilise this presently.

Turning to $\left(g \bar{\psi} \gamma_{5} \psi \phi\right)_{4 D}$ theory, the inverse renormalized $\psi$ propagator to that order reads,

$$
S^{-1}(p)=\gamma \cdot p\left[1-\frac{g^{2}}{32 \pi^{2}} \log \left(-\frac{p^{2}}{\mu^{2}}\right)+\ldots\right],
$$

while the fully off-shell proper vertex part correction is given by

$$
\Lambda_{5}\left(p^{\prime}, p\right)=\left(A+B\left[\gamma \cdot p^{\prime}, \gamma \cdot p\right]\right) \gamma_{5}
$$

where, of the two scalar functions $A$ and $B$, only the former carries the ultraviolet divergence. (A massive theory, apart from modifying $A$ and $B$, would have led to further terms like $\left[C \gamma \cdot p+C^{\prime} \gamma \cdot p^{\prime}\right] \gamma_{5}$.) One readily finds that

$$
A=\frac{i g^{2}}{2} \int \frac{\bar{d}^{2 \ell} k}{k^{2}}\left[\frac{1}{(p+k)^{2}}+\frac{1}{\left(p^{\prime}+k\right)^{2}}-\frac{\left(p-p^{\prime}\right)^{2}}{(p+k)^{2}\left(p^{\prime}+k\right)^{2}}\right]
$$

and

$$
\begin{aligned}
B= & -\frac{i g^{2}}{2 \Delta} \int \frac{\bar{d}^{2 \ell} k}{k^{2}}\left[\left(p^{2}-p^{2}\right)\left(\frac{1}{(p+k)^{2}}-\frac{1}{\left(p^{\prime}+k\right)^{2}}\right)+\left(p-p^{\prime}\right)^{2} \times\right. \\
& \left.\left(\frac{p^{2}+p^{\prime 2}-\left(p-p^{\prime}\right)^{2}}{(p+k)^{2}\left(p^{\prime}+k\right)^{2}}+\frac{1}{(p+k)^{2}}+\frac{1}{\left(p^{\prime}+k\right)^{2}}-\frac{2 k^{2}}{(p+k)^{2}\left(p^{\prime}+k\right)^{2}}\right)\right]
\end{aligned}
$$


to be taken in the limit $\ell \rightarrow 2$. In eq. (10), $\Delta$ is nothing but the Källen function, namely

$$
\Delta \equiv 4\left[\left(p \cdot p^{\prime}\right)^{2}-p^{2} p^{2}\right]=p^{4}+p^{4}+\left(p-p^{\prime}\right)^{4}-2 p^{2} p^{2}-2 p^{2}\left(p-p^{\prime}\right)^{2}-2 p^{\prime 2}\left(p-p^{\prime}\right)^{2}
$$

It is interesting to look at the case $\left(p-p^{\prime}\right)^{2} \rightarrow 0$ in (9) and (10) before worrying about renormalization; one gets

$$
\Lambda_{5} \rightarrow \frac{i g^{2}}{2} \int \frac{\bar{d}^{2 \ell} k}{k^{2}}\left[\left(\frac{1}{\left(p^{\prime}+k\right)^{2}}+\frac{1}{(p+k)^{2}}\right)-\frac{\left[\gamma \cdot p^{\prime}, \gamma \cdot p\right]}{p^{\prime 2}-p^{2}}\left(\frac{1}{\left(p^{\prime}+k\right)^{2}}-\frac{1}{(p+k)^{2}}\right)\right] \gamma_{5}
$$

Again we notice the structure $\left(\Sigma\left(p^{\prime}\right)-\Sigma(p)\right) /\left(p^{\prime 2}-p^{2}\right)$ in the finite part, even though we are not dealing with a gauge theory. Anyhow, renormalizing so that $\Lambda_{5}(p, p)=0$ for $p^{2}=-\mu^{2}$, the finite $B$-type term remains unaffected, and we are left with the lightlike limit

$$
\Gamma_{5} \rightarrow \gamma_{5}-\frac{g^{2}}{32 \pi^{2}}\left[\log \left(\frac{p^{2} p^{2}}{\mu^{4}}\right)+\frac{\ln \left(p^{2} / p^{2}\right)}{p^{2}-p^{\prime 2}}\left[\gamma \cdot p^{\prime}, \gamma \cdot p\right]\right] \gamma_{5}
$$

Our aim is to identify these expressions as first order in $g^{2}$ parts of some nonperturbative construct, so as to capture at the very least the triangular topology of the full vertex function, and then see how far we can take it from there.

\subsection{Nonperturbative form}

Let us start by reducing the perturbative vertex to a more manageable form. The first order expression for $\Lambda\left(p_{1}, p_{2}, p_{3}\right)$ involves hypergeometric functions of two variables when the dimension and masses are arbitrary. However it can be simplified for $m_{i}=0$ in a very elegant way and Davydychev 8] has shown the way to do this: draw three lengths of size $\sqrt{p_{1}^{2}}, \sqrt{p_{2}^{2}}, \sqrt{p_{3}^{2}}$ and let $\Theta_{123}=1$ if a Euclidean triangle can be drawn with those sides, 0 otherwise. Then $\Delta_{E}=-\Delta=2\left(p_{1}^{2} p_{2}^{2}+p_{2}^{2} p_{3}^{2}+p_{3}^{2} p_{1}^{2}\right)-p_{1}^{4}-p_{2}^{4}-p_{3}^{4}$ is four times the square of the area of such a triangle. The internal angles $\phi_{i}$ of the triangle are given in an obvious notation by $2 \sin \phi_{1}=\sqrt{\Delta_{E} / p_{2}^{2} p_{3}^{2}}, 2 \cos \phi_{1}=\left(p_{2}^{2}+p_{3}^{2}-p_{1}^{2}\right) / \sqrt{p_{2}^{2} p_{3}^{2}}$, etc. and of course $\phi_{1}+\phi_{2}+\phi_{3}=\pi$. The result for

$$
\Lambda\left(p_{1}^{2}, p_{2}^{2}, p_{3}^{2}\right)=i g^{2} \int \bar{d}^{2 \ell} r /\left[r^{2}\left(p_{1}-r\right)^{2}\left(p_{2}+r\right)^{2}\right]
$$

which leads to the hypergeometric answer,

$$
\begin{aligned}
\Lambda= & \frac{g^{2} \Gamma(2-\ell)}{(4 \pi)^{\ell}\left(-p_{1}^{2} p_{2}^{2} p_{3}^{2}\right)^{2-\ell}}\left[2 \pi \Delta_{E}^{3 / 2-\ell} \Theta_{123}-\frac{\Gamma^{2}(\ell-1)}{\Gamma(2 \ell-2)} \times\right. \\
& \left.\left(\frac{\left(p_{1}^{2} p_{2}^{2}\right)^{2-\ell}}{p_{1}^{2}+p_{2}^{2}-p_{3}^{2}}{ }_{2} F_{1}\left(1, \frac{1}{2} ; \ell-\frac{1}{2} ;-\frac{\Delta_{E}}{\left(p_{1}^{2}+p_{2}^{2}-p_{3}^{2}\right)^{2}}\right)+2 \text { perms }\right)\right]
\end{aligned}
$$

can be converted into the beautiful form,

$$
\Lambda=\frac{g^{2} \Gamma(2-\ell) \Delta_{E}^{3 / 2-\ell}}{(4 \pi)^{\ell}\left(-p_{1}^{2} p_{2}^{2} p_{3}^{2}\right)^{2-\ell}}\left[2 \pi \Theta_{123}-\frac{\Gamma^{2}(\ell-1)}{\Gamma(2 \ell-3)} \sum_{k=1}^{3} \int_{0}^{2 \phi_{k}} d \chi\left(4 \sin ^{2} \frac{\chi}{2}\right)^{\ell-2}\right] .
$$


Another useful expression (in that limits can be taken more easily) is obtained by adopting an integral representation of the hypergeometric functions; thus

$$
\Lambda=\frac{g^{2} \Gamma(2-\ell) \Delta_{E}^{3 / 2-\ell}}{(4 \pi)^{\ell}\left(-p_{1}^{2} p_{2}^{2} p_{3}^{2}\right)^{2-\ell}}\left[2 \pi \Theta_{123}-\frac{\Gamma^{2}(\ell-1)}{\Gamma(2 \ell-3)} \sum \int_{0}^{1} \frac{\left(p_{i}^{2} p_{j}^{2}\right)^{2-\ell} t^{\ell-5 / 2} d t}{\Delta_{E}^{3 / 2-\ell} \sqrt{4 p_{i}^{2} p_{j}^{2}-\Delta_{E} t}}\right] .
$$

Davydychev has gone further and expressed $\Lambda$ as a series in $(\ell-2)$ leading to polylog functions $\mathrm{Ls}_{j}$, but we shall not require that expansion. Note that the residue at $\ell=2$ of eq.(12) or (13) vanishes (as it must since the vertex is convergent in 4-D), and that the residue in 6 - $\mathrm{D}$ at $\ell=3$ reduces to $-g^{2} / 128 \pi^{3}$, because of the identity,

$$
p_{3}^{2}\left(p_{1}^{2}+p_{2}^{2}-p_{3}^{2}\right)+p_{1}^{2}\left(p_{2}^{2}+p_{3}^{2}-p_{1}^{2}\right)+p_{2}^{2}\left(p_{3}^{2}+p_{1}^{2}-p_{2}^{2}\right)=\Delta_{E}
$$

Another interesting situation arises when $p_{1}^{2} \equiv p^{2}, p_{2}^{2} \equiv p^{\prime 2}, p_{3}^{2}=0$, whereupon $\Delta_{E}=-\left(p^{2}-p^{2}\right)^{2}, \tan ^{2} \phi_{1}=-1, \tan ^{2} \phi_{2}=-1, \tan ^{2} \phi_{3}=\left(p^{2}-p^{\prime}\right)^{2} /\left(p^{2}+p^{2}\right)^{2}, \Theta_{123}=0$. Thus

$$
\Lambda\left(p^{2}, p^{\prime 2}, 0\right)=\frac{16 g^{2} \Gamma(2-\ell) \Gamma(1 / 2)}{(16 \pi)^{\ell} \Gamma(\ell-3 / 2)} \frac{\left[\left(-p^{\prime 2}\right)^{\ell-2}-\left(-p^{2}\right)^{\ell-2}\right]}{\left[p^{\prime 2}-p^{2}\right]},
$$

which allows one to take the limit $p^{2}=p^{2} \rightarrow-\mu^{2}$, namely

$$
\Lambda\left(-\mu^{2},-\mu^{2}, 0\right)=\frac{16 g^{2} \Gamma(3-\ell) \Gamma(\ell-2) \Gamma(1 / 2) \mu^{2 \ell-6}}{(16 \pi)^{\ell} \Gamma(\ell-3 / 2)} \rightarrow-\frac{g^{2}}{2(4 \pi)^{3}(\ell-3)},
$$

as $\ell \rightarrow 3$.

We now suggest a way of "going nonperturbative", which captures the essence of the vertex triangular topology. We firstly observe that the asymptotic form of the inverse propagator $\Delta_{\phi}^{-1}(p) \simeq\left(p^{2}\right)^{1+\gamma_{\phi}}$ can be gotten directly from the self-energy $\Sigma(p)$ in $2 \ell$ dimensions simply be making the replacement $\ell=3+\gamma_{\phi}$ in the dimensionally continued result, apart from an overall factor that must be carefully chosen to accord with the renormalization condition. This procedure will lead from $(6)$ to $\Delta_{\phi}^{-1}(p)=p^{2}\left(-p^{2} / \mu^{2}\right)^{\gamma_{\phi}}$, with $\gamma_{\phi}=-g^{2} / 6(4 \pi)^{3}$ to first order.

Applying the same procedure to the $\left(\phi^{\dagger} \phi \sigma\right)_{6 D}$ vertex, we will end up with

$\Gamma\left(p_{1}, p_{2}, p_{3}\right)=\frac{p_{1}^{2} p_{2}^{2} p_{3}^{2}}{\Delta_{E}^{3 / 2}}\left(\frac{p_{1}^{2} p_{2}^{2} p_{3}^{2}}{\mu^{2} \Delta_{E}}\right)^{\gamma_{\Gamma}}\left[2 \pi \Theta_{123}-\frac{\Gamma^{2}\left(2+\gamma_{\Gamma}\right)}{\Gamma\left(3+2 \gamma_{\Gamma}\right)} \sum_{k=1}^{3} \int_{0}^{2 \phi_{k}} d \chi\left(4 \sin ^{2} \frac{\chi}{2}\right)^{1+\gamma_{\Gamma}}\right]$,

where $\gamma_{\Gamma}=-g^{2} / 2(4 \pi)^{3}$ to first order. Realising that $\sum_{k} \phi_{k}=\pi$ and

$$
\sum_{k=1}^{3} \sin \left(2 \phi_{k}\right)=\Delta_{E}^{3 / 2} / 2 p_{1}^{2} p_{2}^{2} p_{3}^{2}
$$

we can readily establish that $\Gamma \rightarrow 1$ when $\gamma_{\Gamma} \rightarrow 0$. Also one may check that an expansion of (15) to order $g^{2}$ reproduces perturbation theory, including terms like

$$
\sum_{k=1}^{3} \int_{0}^{2 \phi_{k}} d \chi\left(4 \sin ^{2} \frac{\chi}{2}\right) \log \left(4 \sin ^{2} \frac{\chi}{2}\right)
$$


which appear after renormalization. Thus we are emboldened to regard (15) as a decent nonperturbative vertex that incorporates triangular topological contributions, but whether we can easily make use of it is entirely another matter since its form is analytically complicated. Perhaps a more amenable form of (15) is

$\Gamma\left(p_{1}^{2}, p_{2}^{2}, p_{3}^{2}\right)=\frac{\left(p_{1}^{2} p_{2}^{2} p_{3}^{2}\right)^{1+\gamma_{\Gamma}}}{\mu^{2 \gamma_{\Gamma}} \Delta_{E}^{3 / 2+\gamma_{\Gamma}}} \frac{\Gamma\left(3+2 \gamma_{\Gamma}\right)}{\Gamma^{2}\left(2+\gamma_{\Gamma}\right)}\left[2 \pi \Theta_{123}-\sum_{k} \frac{\left(\sin \phi_{k}\right)^{3+2 \gamma_{\Gamma}}}{\cos \phi_{k}} \int_{0}^{1} \frac{(1-t)^{1+\gamma_{\Gamma}} d t}{\sqrt{t}\left(1+t \tan ^{2} \phi_{k}\right)}\right]$,

because, without too much trouble, it allows us to take the lightlike limit,

$$
\Gamma\left(p^{2}, p^{\prime 2}, 0\right)=\frac{\mu^{2}}{\left(1+\gamma_{\Gamma}\right)\left(p^{2}-p^{2}\right)}\left[\left(-\frac{p^{2}}{\mu^{2}}\right)^{1+\gamma_{\Gamma}}-\left(-\frac{p^{\prime 2}}{\mu^{2}}\right)^{1+\gamma_{\Gamma}}\right],
$$

a result which we foresaw earlier. The special limit when one leg carries zero momentum, $\Gamma\left(p^{2}, p^{2}, 0\right)=\left(-p^{2} / \mu^{2}\right)^{\gamma_{\Gamma}}$ is then readily found. Also the renormalized perturbative answer stated at the end of the last subsection falls out upon expansion to first order in $\gamma_{\Gamma}$, provided the anomalous dimension is correctly identified.

Let us perform a similar procedure on $\left(\bar{\psi} \gamma_{5} \phi \psi\right)_{4 D}$. Here the full vertex, consists of two terms:

$$
\Gamma_{5}\left(p^{\prime}, p\right)=\left(\Gamma_{A}+\Gamma_{B}\left[\gamma \cdot p^{\prime}, \gamma \cdot p\right]\right) \gamma_{5}
$$

whose first order in $g^{2}$ terms are summarised in (9) and (10). In making the substitution $\ell \rightarrow 2+\gamma$ so as to obtain a nonperturbative expression, it is very easy to handle the propagator (7) and arrive at

$$
S^{-1}(p)=\gamma \cdot p\left(-p^{2} / \mu^{2}\right)^{\gamma_{\psi}} ; \quad \gamma_{\psi}=-g^{2} / 32 \pi^{2} .
$$

Also it is possible to exponentiate the (renormalized) self-energy like terms in the vertex parts $A$ and $B$ arising in eqs (9) and (10):

$$
1+i g^{2} \int \bar{d}^{2 \ell} k / k^{2}(p+k)^{2} \rightarrow 1+\left(g^{2} / 16 \pi^{2}\right) \log \left(-p^{2} / \mu^{2}\right) \rightarrow\left(-p^{2} / \mu^{2}\right)^{g^{2} / 16 \pi^{2}}
$$

On the other hand the full triangular topology integral (12) or (13) will produces a finite result

$$
\Gamma_{F}\left(p_{1}^{2}, p_{2}^{2}, p_{3}^{2}\right)=\frac{4}{\sqrt{\Delta_{E}}}\left(\frac{-p_{1}^{2} p_{2}^{2} p_{3}^{2}}{\mu^{2} \Delta_{E}}\right)^{\gamma_{\Gamma}}\left[2 \pi \Theta_{123}-\frac{\Gamma^{2}\left(1+\gamma_{\Gamma_{E}}\right)}{\Gamma\left(1+2 \gamma_{\Gamma_{E}}\right)} \sum_{k} \int_{0}^{2 \phi_{k}} d \theta\left(\sin ^{2} \theta\right)^{-\gamma_{\Gamma}}\right] .
$$

One may verify that the vertex scaling behaviour is reproduced in $\Gamma_{F}$ and that it vanishes for $\gamma_{\Gamma}=0$, as it should in zeroth order. Anyhow, combining the terms, we arrive at the nonperturbative Yukawa vertex parts,

$$
\begin{aligned}
& 2 \Gamma_{A}\left(p^{\prime}, p\right)=\left(-p^{2} / \mu^{2}\right)^{\gamma_{\Gamma}}+\left(-p^{2} / \mu^{2}\right)^{\gamma_{\Gamma}}-\left(p-p^{\prime}\right)^{2} \Gamma_{F} \\
& \Delta \Gamma_{B}\left(p^{\prime}, p\right)=\left(p^{2}-p^{2}\right)\left[\left(-p^{\prime 2} / \mu^{2}\right)^{\gamma_{\Gamma}}-\left(-p^{2} / \mu^{2}\right)^{\gamma_{\Gamma}}\right]+\left(p-p^{\prime}\right)^{2} \Gamma_{F B},
\end{aligned}
$$

where $\Gamma_{F B}=\left[\left(p-p^{\prime}\right)^{2}-p^{2}-p^{\prime 2}\right] \Gamma_{F}+2\left(-\left(p-p^{\prime}\right)^{2} / \mu^{2}\right)^{\gamma_{\Gamma}}-\left(-p^{2} / \mu^{2}\right)^{\gamma_{\Gamma}}-\left(-p^{2} / \mu^{2}\right)^{\gamma_{\Gamma}}$. 


\section{Applications}

The question is how to apply all this. One's first inclination is to substitute the vertex (15) or (16) into (1) and (2) so as to find the relation between anomalous dimension and coupling constant - a relation which is normally found by a tedious process of perturbative renormalization and is worked out to order $g^{6}$ at least. Our proposal is that nonperturbative forms of propagator and vertex avoid the need for renormalization since the skeleton expansion is automatically regularized at the physical dimension $D=4$ or 6; as we shall see, such a procedure will lead to a transcendental relation between $\gamma$ and

$g^{2}$. This happens even when one truncates to the first contribution to the self-energy, having the form

$$
\int \Gamma(p, p+k) \Delta(p+k) \Delta(k) \Gamma(p+k, p) d^{D} k
$$

although one ought properly to consider the entire series of skeleton terms with their ever more intricated topologies (and matching vertices). Nonetheless, considering even the first term of the skeleton series is a substantial improvement on past efforts [3, 4] and is worthy of study.

The task of evaluating the first term $g^{2} F_{2}\left(p, \mu, \gamma\left(g^{2}\right)\right)$ is rather daunting: one is required to integrate the product of two dressed propagators with the square of expression (15) or expression (16), and this is technically very demanding. Numerical calculations are useless from that viewpoint because one is interested in obtaining the analytical connection between $\gamma$ and $g^{2}$, even if comparison with perturbation theory eventually necessitates a power series expansion in $g^{2}$. A number of helpful auxiliary integrals are collected in the appendix, but we are forced to admit that the full-blown integration producing $F_{2}$ is presently beyond our technical reach. Faute de mieux we are forced to approximate the skeleton Feynman integral by one which is doable and which captures the essence of the idea: the main thing is to ensure that the non-perturbative vertex has the correct scaling behaviour, symmetry properties and analytic behaviour as far as possible, and that it should by itself regularize the intermediate momentum integral.

In the following we shall attempt to use a few approximations to the nonperturbative triangular vertex that reflect its main features, in order to extract the relation between anomalous dimension and coupling constant. Thus we will examine a number of models in which $F_{2}$ is free of infinities and is automatically regularized it all too easy to construct models which have the correct scaling property but which nevertheless contain infinities - but where the vertex singularities are not quite correct.

\subsection{Model 1 for $\left(\phi^{\dagger} \phi \sigma\right)_{6 D}$}

Here we make the choice $\Gamma(p, p+k, k)=\left(p^{2}(p+k)^{2} / \mu^{2} k^{2}\right)^{\gamma}$ and leave the $\sigma$ propagator undressed. This has the virtue of simplicity; it possesses symmetry at the $\phi$ legs and correct scaling but is otherwise awry in its analytical properties and especially its vertex singularities. Ignoring these defects, and using the results in the Appendix, we obtain 
the self-consistency relation

$$
p^{2(1+\gamma)}=i g^{2} \int \frac{\bar{d}^{6} k}{k^{2}(p+k)^{2(1+\gamma)}}\left(\frac{p^{2}(p+k)^{2}}{k^{2}}\right)^{2 \gamma},
$$

or

$$
1=a \frac{\Gamma(-1+\gamma) \Gamma(2-2 \gamma) \Gamma(2+\gamma)}{\Gamma(4-\gamma) \Gamma(1+2 \gamma) \Gamma(1-\gamma)} ; \quad a \equiv \frac{g^{2}}{(4 \pi)^{3}}
$$

This may be contrasted with the rainbow approximation where the self-consistency relation instead reads,

$$
p^{4} \Delta(p)=-i g^{2} \int \Delta(p+k) \bar{d}^{6} k / k^{2} \quad \text { and } \quad 1=a / \gamma(\gamma-1)(\gamma-2)(\gamma-3) .
$$

To obtain a perturbative expansion of (21) we take a series in $\gamma$ or $a$ as needed to arrive at

$$
\gamma_{\text {model } 1}=-\frac{a}{6}+\frac{11 a^{2}}{6^{3}}-\frac{134 a^{3}}{6^{5}}+\cdots
$$

compared with $[\underline{5}$

$$
\gamma_{\text {rainbow }}=-\frac{a}{6}+\frac{11 a^{2}}{6^{3}}-\frac{206 a^{3}}{6^{3}}+\cdots, \quad \gamma_{\text {chain }}=-\frac{a}{6}+\frac{11 a^{2}}{6^{3}}-\frac{170 a^{3}}{6^{3}}+\cdots
$$

\subsection{Model 2 for $\left(\phi^{\dagger} \phi \sigma\right)_{6 D}$}

We now consider a vertex which better captures the analytical behaviour of the triangular topology but which is necessarily more complicated than the previous model. Here we try to mimic some of the dependence on $\tan ^{2} \phi_{i}$ which arises in (11) by letting

$\Gamma^{2}\left(p_{1}^{2}, p_{2}^{2}, p_{3}^{2}\right)=\left[\left(p_{1}^{2}\right)^{1+2 \gamma}\left(p_{2}^{2}+p_{3}^{2}-p_{1}^{2}\right)+\left(p_{2}^{2}\right)^{1+2 \gamma}\left(p_{3}^{2}+p_{1}^{2}-p_{2}^{2}\right)+p_{3}^{2}\left(p_{1}^{2} p_{2}^{2}\right)^{\gamma}\left(p_{1}^{2}+p_{2}^{2}-p_{3}^{2}\right)\right] / \Delta_{E}$.

Making use of the equations in the appendix, we may arrive at the self-consistency relation,

$$
\begin{aligned}
1 & =\frac{a}{6}\left[\frac{1}{(2-\gamma)(\gamma-1)}-\frac{1}{\gamma(1-\gamma)}+\frac{1}{(2+\gamma)(3+\gamma)}-\frac{1}{(1+\gamma)(2+\gamma)}\right] \\
& =-\frac{a}{6}\left[\frac{1}{\gamma}+\frac{11}{6}+\frac{41 \gamma}{36}+\cdots\right] .
\end{aligned}
$$

This corresponds to the series

$$
\gamma_{\text {model } 2}=-\frac{a}{6}+\frac{11 a^{2}}{6^{3}}-\frac{162 a^{3}}{6^{5}}+\cdots
$$

\subsection{Model 3 for $\left(\bar{\psi} \gamma_{5} \psi \phi\right)_{4 D}$}

Before making any approximations, we may note that the $F_{2}$ contribution to the inverse fermion propagator $\gamma \cdot p S^{-1}(p)$, with

$$
\Gamma_{5}=\left(\Gamma_{A}+\Gamma_{B}\left[\gamma \cdot p, \gamma \cdot p^{\prime}\right]\right) \gamma_{5}
$$


can be written as

$$
i \frac{g^{2}}{p^{2}} \int \frac{\bar{d}^{4} k}{\left((p+k)^{2}\right)^{1+\gamma}} \Delta_{\sigma}(k)\left[\frac{1}{2}\left(p^{2}+(p+k)^{2}-k^{2}\right)\left(\Gamma_{A}^{2}-\Delta_{E} \Gamma_{B}^{2}\right)+\Delta_{E} \Gamma_{A} \Gamma_{B}\right] .
$$

Therefore, if we use a quenched $\sigma$ approximation, the $F_{2}$ term leads to the following self-consistent relation for the anomalous dimension,

$$
\left(p^{2}\right)^{1+\gamma}=i g^{2} \int \frac{\bar{d}^{4} k}{2 k^{2}\left((p+k)^{2}\right)^{1+\gamma}}\left[\left(p^{2}+(p+k)^{2}-k^{2}\right)\left(\Gamma_{A}^{2}-\Delta_{E} \Gamma_{B}^{2}\right)+2 \Delta_{E} \Gamma_{A} \Gamma_{B}\right]
$$

into which we may feed various models for the vertex parts $\Gamma_{A, B}$. Of course we would dearly have loved to make use of their nonperturbative forms given in the previous section (consistent with triangular topology) but find the resulting computation too hard analytically; so we are obliged to model something resembling the true vertex that is within our capabilities. Noting expressions (9) and (10) and ensuring correct scaling, we take

$$
\Gamma_{A}=\left(\frac{p^{2}(p+k)^{2}}{\mu^{2} k^{2}}\right)^{\gamma} \text { and } \quad \Delta_{E} \Gamma_{B}=\gamma\left[p^{2}-(p+k)^{2}\right]\left(\frac{p^{2} k^{2}}{\mu^{2}(p+k)^{2}}\right)^{\gamma} .
$$

Inserting this into (24) we end up with

$$
1=-\frac{a}{2 \gamma}\left[1+\frac{5 \gamma}{2}+\frac{13 \gamma^{2}}{4}+\cdots\right], \quad a \equiv \frac{g^{2}}{16 \pi^{2}}
$$

corresponding to

$$
\gamma_{\text {model } 3}=-\frac{a}{2}+\frac{a^{2}}{2^{3}}-\frac{14 a^{2}}{2^{5}}+\cdots
$$

This result should be compared with the rainbow and chain approximations [5] which, for Yukawa theory, read

$$
\gamma_{\text {rainbow }}=-\frac{a}{2}+\frac{a^{2}}{2^{3}}-\frac{2 a^{2}}{2^{5}}+\cdots, \quad \gamma_{\text {chain }}=-\frac{a}{2}+\frac{a^{2}}{2^{3}}-\frac{2 a^{2}}{2^{5}}+\cdots
$$

\subsection{Lightlike Model 4 for $\left(\phi^{\dagger} \phi \sigma\right)_{6 D}$}

We saw earlier that in the lightlike limit of one of the momenta, not necessarily the limit of zero momentum transfer, the vertex function assumed the form of the difference of two self-energies, at least to first order in perturbation theory. Let us therefore make the approximation

$$
\Delta_{\phi}(p+k) \Gamma(p+k, p) \Delta_{\phi}(p) \simeq \frac{\Delta_{\phi}(p+k)-\Delta_{\phi}(p)}{p^{2}-(p+k)^{2}}
$$

in quenched $\left(\phi^{\dagger} \phi \sigma\right)_{6 D}$ theory, since we have already disregarded the $\sigma$ field dressing. Using the usual spectral form of the $\phi$ field propagator, we may therefore substitute

$\Delta_{\phi}(p)=\int_{0}^{\infty} \frac{\rho\left(w^{2}\right) d w^{2}}{p^{2}-w^{2}}, \quad \Delta_{\phi}(p+k) \Gamma(p+k, p) \Delta_{\phi}(p) \simeq \int_{0}^{\infty} \frac{\rho\left(w^{2}\right) d w^{2}}{\left((p+k)^{2}-w^{2}\right)\left(p^{2}-w^{2}\right)}(26)$ 
in the Schwinger-Dyson equation,

$$
Z_{\phi}^{-1}=p^{2} \Delta_{\phi}(p)-i g^{2} Z_{\phi}^{-1} Z_{g} \int \bar{d}^{6} k \Delta_{\phi}(p+k) \Gamma(p+k, p) \Delta_{\phi}(p) / k^{2} .
$$

Recalling that $Z_{\phi}^{-1}=\int \rho\left(w^{2}\right) d w^{2}$, the spectral equation reduces to

$$
\int d w^{2} \rho\left(w^{2}\right) \frac{w^{2} Z_{\phi} Z_{g}^{-1}+\Sigma(p, w)}{p^{2}-w^{2}}=0
$$

where $\Sigma(p, w)=g^{2} \int \bar{d}^{2 \ell} k /\left[k^{2}\left((p+k)^{2}-w^{2}\right)\right]$ is the first order self-energy for a $\phi$ field of mass $w$, to be taken in the limit as $\ell \rightarrow 3$. This has essentially the same form as in the gauge technique 9] for QED, except that it is no longer true that $Z_{g}=Z_{\phi}$ - and this is just what one needs! The point is that the self-energy carries the infinity (in its real part) leaving us with the representation

$$
\Sigma(p, w)=\frac{a}{\ell-3}\left[\frac{1}{6} p^{2}-\frac{1}{2} w^{2}\right]+\frac{\left(p^{2}-w^{2}\right)^{2}}{\pi} \int \frac{\Im \Sigma(s, w) d s}{\left(s-w^{2}\right)^{2}\left(s-p^{2}-i \epsilon\right)},
$$

while $Z_{\phi}^{-1} Z_{g}=1+2 a /(3(\ell-3)$ and they both combine neatly to produce a factor $\left(p^{2}-w^{2}\right)$ in the numerator of (28). Therefore taking the imaginary part of (28), one arrives at

$$
-\pi p^{2} \rho\left(p^{2}\right)+\int \frac{\Im \Sigma(p, w) \rho\left(w^{2}\right) d w^{2}}{p^{2}-w^{2}}=0 .
$$

Since $\Im \Sigma(p, w)=g^{2}\left(p^{2}-w^{2}\right)^{2} / 6(4 \pi)^{3} p^{4}, \quad$ or $\quad \Im \Sigma(p, w) /\left(p^{2}-w^{2}\right)=a\left(1-w^{2} / p^{2}\right)^{2} / 6$, (29) may be solved by use the ansatz $\rho\left(w^{2}\right) \propto\left(w^{2}\right)^{-1-\gamma}$, yielding the sought-after relation

$$
1=-\frac{a}{3 \gamma(1-\gamma)(2-\gamma)} \quad \text { or } \quad \gamma_{\text {model } 4}=-\frac{a}{6}+\frac{9 a^{2}}{6^{3}}-\frac{144 a^{3}}{6^{5}} \ldots
$$

only exact to order $a$. Evidently $\Delta_{\phi}(p)$ has the same anomalous dimension as its spectral function (or imaginary part) $\rho$.

All the above model conclusions should be treated with great caution and some scepticism. We supplied models of the true vertex whose scaling coincided to second order with known results for the anomalous dimension, but which possessed incorrect singularities. It would have been quite easy to change radically the results of our calculations by inputting equally plausible vertex ansätze. So all we can purport to have demonstrated to the critical reader is that it is possible, in principle, to regulate the skeleton expansion by using nonperturbative propagators and vertices all the while staying in integer dimensions. In the end we dare only claim that the scheme outlined in the introduction is a viable method for discovering the anomalous scaling properties of the field theory in question. After all, the skeleton expansion has recently proved its worth in a similar context [10] and we have no reason to suspect that it will fail us in the present circumstances. This said we are at a computational impasse in our approach in attempting to include the proper vertex with its full complement of singularities; unfortunately we see no easy way out of this difficulty if we shy away from numerical methods. 


\section{Acknowledgments}

We thank the Australian Research Council for providing financial support for this project, under grant number A00000780. We also benefitted from conversations with Dirk Kreimer and M Kalmykov.

\section{Appendix}

Here we present a number of auxiliary integrals which assist in determining the connection between $\gamma$ and $g^{2}$. The first one of these is

$$
I_{a b c} \equiv-i \int \bar{d}^{2 \ell} k /\left(k^{2}\right)^{a}\left((p+k)^{2}\right)^{b} \Delta_{E}^{c} ; \quad \Delta_{E}=4\left[k^{2} p^{2}-(k \cdot p)^{2}\right] .
$$

The case $c=0$ is rather well-known to practioners in this field and can be found, using Feynman parametric techniques, to equal

$$
I_{a b 0}=\frac{\left(p^{2}\right)^{\ell-a-b}}{(-4 \pi)^{\ell}} \frac{\Gamma(a+b-\ell) \Gamma(\ell-a) \Gamma(\ell-b)}{\Gamma(a) \Gamma(b) \Gamma(2 \ell-a-b)} .
$$

For $c \neq 0$ we must resort to another method in order to find $I_{a b c}$. Namely we go to the frame where $p=i\left(\sqrt{q^{2}} ; \overrightarrow{0}\right) ; q^{2} \equiv-p^{2}, k=\sqrt{K^{2}}(i \cos \theta ; \sin \theta, \ldots) ; K^{2} \equiv-k^{2}$, so

$$
\begin{gathered}
\vec{d}^{2 \ell} k=i \frac{K^{2 \ell-1} d K}{(2 \pi)^{2 \ell}} \cdot(\sin \theta)^{2 \ell-2} d \theta \cdot \frac{2 \pi^{\ell-1 / 2}}{\Gamma(\ell-1 / 2)}, \\
(p+k)^{2}=-q^{2}-K^{2}-2 \sqrt{q^{2} K^{2}} \cos \theta ; \quad \Delta_{E}=4 K^{2} q^{2} \sin ^{2} \theta .
\end{gathered}
$$

Hence

$$
I_{a b c}=\int_{0}^{\infty} \frac{2 \pi^{\ell-1 / 2} K^{2 \ell-1} d K}{(2 \pi)^{2 \ell} \Gamma(\ell-1 / 2)} \int_{0}^{\pi} \frac{(\sin \theta)^{2 \ell-2} d \theta}{\left(-K^{2}\right)^{a}\left(-q^{2}-K^{2}-2 q K \cos \theta\right)^{b}\left(4 K^{2} q^{2} \sin ^{2} \theta\right)^{c}} .
$$

But the standard texts inform us that

$$
\int_{0}^{\pi} \frac{(\sin \theta)^{\beta-1} d \theta}{\left(1+2 z \cos \theta+z^{2}\right)^{\alpha}}=\sqrt{\pi} \Gamma(\beta) F\left(\alpha, \alpha-\beta+1 / 2 ; \beta+1 / 2 ; z^{2}\right) / \Gamma(\beta+1 / 2)
$$

and

$$
\int_{0}^{\infty} K^{2 \sigma-1} F\left(\alpha, \beta ; \gamma ; \frac{q^{2}}{K^{2}}\right) d K=\left(-q^{2}\right)^{\sigma} \frac{\Gamma(\gamma) \Gamma(-\sigma) \Gamma(\alpha+\sigma) \Gamma(\beta+\sigma)}{2 \Gamma(\alpha) \Gamma(\beta) \Gamma(\gamma+\sigma)} .
$$

Putting this all together we end up with

$$
I_{a b c}=\frac{\left(p^{2}\right)^{\ell-a-b-2 c}}{(-4 \pi)^{\ell} 4^{c}} \frac{\Gamma(\ell-c-1 / 2)}{\Gamma(\ell-1 / 2)} \frac{\Gamma(a+b+c-\ell) \Gamma(\ell-a-b) \Gamma(\ell-b-c)}{\Gamma(a) \Gamma(b) \Gamma(2 \ell-a-b-2 c)} .
$$

In particular, for $6 \mathrm{D}$, the result reads

$$
I_{a b 1}=\frac{\left(p^{2}\right)^{1-a-b}}{6(-4 \pi)^{3}} \frac{\Gamma(a+b-2) \Gamma(2-a) \Gamma(2-b)}{\Gamma(a) \Gamma(b) \Gamma(4-a-b)} .
$$

Therefore if one takes $b$ non-integer for the present, the limit as $a \rightarrow 0,-1$ will produce a vanishing result leading to $I_{0 b 1}=I_{-1 b 1}=0$. We take this result to be correct even for integer $b$, paralleling the treatment of tadpole integrals in dimensional regularization. 


\section{References}

[1] Mack G and Todorov IT 1973, Phys. Rev. D6 1764

[2] Palchik MYa and Fradkin ES 1999, Dokl. Phys. 44754

Zaikin VN and Palchik MYa 2001, Theor. Math. Phys. 1281181

[3] Delbourgo R, Kalloniatis A and Thompson G 1996, Phys. Rev. D54 1095

Delbourgo R, Elliott D and McAnally D 1997, Phys. Rev. D55 5230

[4] Fukuda R and Kugo T 1976, Nucl. Phys. B117 250

Higashijima K 1984, Phys. Rev. D29 1228

Roberts CD and Williams AG 1994, Prog. Part. Nucl. Phys. 33477

[5] Broadhurst DJ and Kreimer D 1999, J. Symb. Comput. 27581

Broadhurst DJ and Kreimer D 2001, Nucl. Phys. B600 403

[6] Macfarlane AJ and Woo G 1974, Nucl. Phys. B77 91

[7] Boos EE and Davydychev AI 1991, Theor. Math. Phys. 891052

Davydychev AI 1992, J. Phys. 25A 5587

[8] Davydychev AI 2000, Phys. Rev. D61 087701

[9] Delbourgo R 1979, Nuovo Cim. 49A 484

[10] Broadhurst DJ, Delbourgo R and Kreimer D 1996, Phys. Lett. B366 421 